\title{
Photon-noise limited sensitivity in titanium nitride kinetic inductance detectors
}

J. Hubmayr', J. Beall, D. Becker, H.-M. Cho, M. Devlin, B. Dober, C. Groppi, G. C. Hilton, K. D. Irwin, D. Li, P. Mauskopf, D. P. Pappas, J. Van Lanen, M. R. Vissers, Y. Wang, L. F. Wei, and J. Gao

Citation: Appl. Phys. Lett. 106, 073505 (2015); doi: 10.1063/1.4913418

View online: http://dx.doi.org/10.1063/1.4913418

View Table of Contents: http://aip.scitation.org/toc/apl/106/7

Published by the American Institute of Physics

\section{Articles you may be interested in}

Titanium nitride films for ultrasensitive microresonator detectors

Applied Physics Letters 97, 102509 (2010); 10.1063/1.3480420

Anomalous response of superconducting titanium nitride resonators to terahertz radiation

Applied Physics Letters 105, 192601 (2014); 10.1063/1.4901536

Operation of a titanium nitride superconducting microresonator detector in the nonlinear regime

Journal of Applied Physics 113, 104501 (2013); 10.1063/1.4794808

Proximity-coupled Ti/TiN multilayers for use in kinetic inductance detectors

Applied Physics Letters 102, 232603 (2013); 10.1063/1.4804286

Low loss superconducting titanium nitride coplanar waveguide resonators

Applied Physics Letters 97, 232509 (2010); 10.1063/1.3517252

Frequency-tunable superconducting resonators via nonlinear kinetic inductance

Applied Physics Letters 107, 062601 (2015); 10.1063/1.4927444

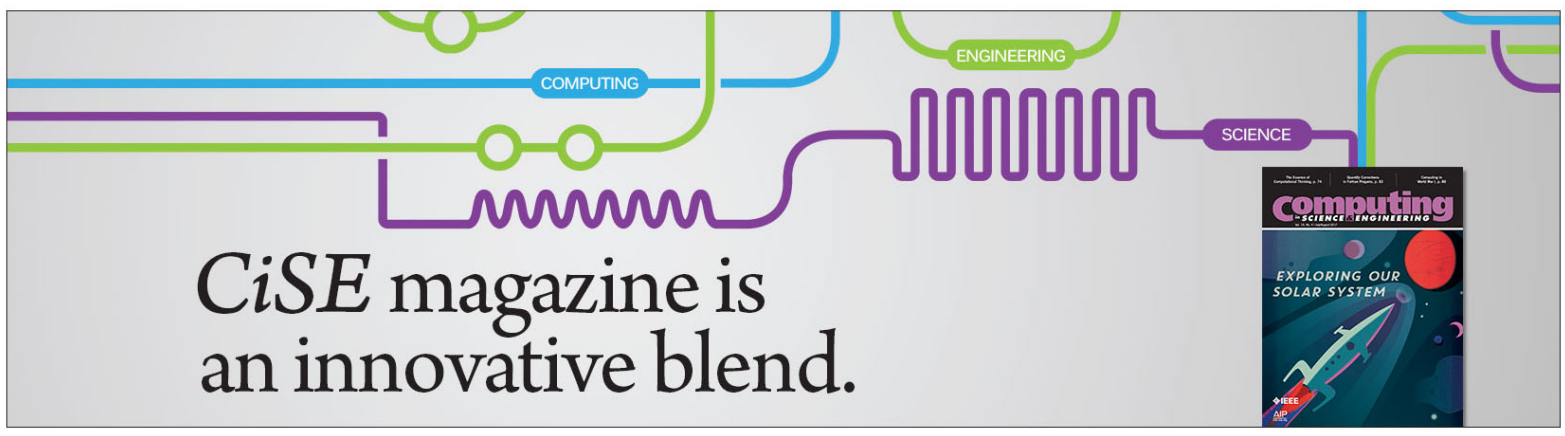




\title{
Photon-noise limited sensitivity in titanium nitride kinetic inductance detectors
}

\author{
J. Hubmayr, ${ }^{1, a)}$ J. Beall, ${ }^{1}$ D. Becker, ${ }^{1}$ H.-M. Cho, ${ }^{1}$ M. Devlin, ${ }^{2}$ B. Dober, ${ }^{2}$ C. Groppi, ${ }^{3}$ \\ G. C. Hilton, ${ }^{1}$ K. D. Irwin, ${ }^{4}$ D. Li, ${ }^{1}$ P. Mauskopf, ${ }^{3}$ D. P. Pappas, ${ }^{1}$ J. Van Lanen, ${ }^{1}$ \\ M. R. Vissers, ${ }^{1}$ Y. Wang, ${ }^{1,5}$ L. F. Wei, ${ }^{5}$ and J. Gao ${ }^{1}$ \\ ${ }^{1}$ National Institute of Standards and Technology, 325 Broadway, Boulder, Colorado 80305, USA \\ ${ }^{2}$ Department of Physics and Astronomy, University of Pennsylvania, 209 South 33rd St., Philadelphia, \\ Pennsylvania 19104, USA \\ ${ }^{3}$ School of Earth and Space Exploration, Arizona State University, 781 S Terrace Rd., Tempe, Arizona 85281, \\ USA \\ ${ }^{4}$ Department of Physics, Stanford University, Stanford, California 94305, USA \\ ${ }^{5}$ Quantum Optoelectronics Laboratory, Southwest Jiaotong University, Chengdu, China
}

(Received 25 June 2014; accepted 24 January 2015; published online 20 February 2015)

\begin{abstract}
We demonstrate photon-noise limited performance at sub-millimeter wavelengths in feedhorncoupled, microwave kinetic inductance detectors made of a TiN/Ti/TiN trilayer superconducting film, tuned to have a transition temperature of $1.4 \mathrm{~K}$. Micro-machining of the silicon-on-insulator wafer backside creates a quarter-wavelength backshort optimized for efficient coupling at $250 \mu \mathrm{m}$. Using frequency read out and when viewing a variable temperature blackbody source, we measure device noise consistent with photon noise when the incident optical power is $>0.5 \mathrm{pW}$, corresponding to noise equivalent powers $>3 \times 10^{-17} \mathrm{~W} / \sqrt{\mathrm{Hz}}$. This sensitivity makes these devices suitable for broadband photometric applications at these wavelengths. (C) 2015 AIP Publishing LLC.

[http://dx.doi.org/10.1063/1.4913418]
\end{abstract}

Microwave kinetic inductance detectors (MKIDs) are superconducting pair breaking devices ${ }^{1}$ currently in development for a broad range of applications. ${ }^{2}$ Making use of high quality factor resonators, MKIDs multiplex in the frequency domain and thus scale to large-format arrays. MKIDs are the implemented or planned detector technology for several submillimeter instruments. $^{3-7}$

The fundamental limit of the sensitivity of a submillimeter photon integrating detector is set by the photon fluctuations from the source under observation, which is referred to as the photon-noise limit or background limit. The noise equivalent power (NEP) of photon fluctuations from a narrow bandwidth source can be expressed as ${ }^{8}$

$$
N E P_{\text {photon }}=\sqrt{\frac{2 P h \nu(1+m \eta)}{\eta}} .
$$

Here, and throughout this letter, we refer NEP terms to the input of an optical system with optical efficiency $\eta$. The first term describes Poisson shot noise. $P$ is the input optical power, $h$ is Planck's constant, and $\nu$ is the observation center frequency. The second term describes photon bunching, where $m$ is the occupation number per mode. MKID detectors are also limited by generation-recombination noise. , $^{9}$ In the limit of photon dominated quasiparticle production, only the recombination noise need be considered, and the NEP can be expressed as ${ }^{11}$

$$
N E P_{R}=\sqrt{\frac{2 P \Delta / \eta_{p b}}{\eta}} .
$$

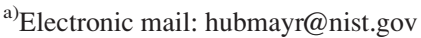

Here, $\Delta$ is the superconducting energy gap and $\eta_{p b} \lesssim 0.57$ is the efficiency of converting photons to quasiparticles. ${ }^{12}$ In this work, since $h \nu \sim 22 \Delta$, the recombination noise is subdominant to the photon-noise.

Recently, the sensitivity of MKIDs has improved. Laboratory studies have shown photon-noise limited performance in aluminum MKIDs ${ }^{13,14}$ as well as TiN-based MKIDs. ${ }^{15}$ As the superconducting material in kinetic inductance detectors, TiN has a number of potential advantages including low loss, ${ }^{16,17}$ high resistivity, large kinetic inductance fraction, and a tunable transition temperature. The spatial non-uniformity of TiN films with $T_{c}<4 \mathrm{~K}$ has been improved by use of a new superconducting film, a TiN/Ti/ TiN trilayer. ${ }^{18}$ These films show $<1 \% T_{c}$ variation across a $75 \mathrm{~mm}$ diameter wafer.

In this work, we report a measurement of photon-noise limited sensitivity at $250 \mu \mathrm{m}$ in kinetic inductance detectors fabricated from TiN/Ti/TiN films at power loads relevant for balloon-borne or satellite-based photometry $(1<P<20$ $\mathrm{pW})$. These polarization-sensitive detectors are under development for the next-generation BLAST experiment. ${ }^{19}$ The $1.4 \mathrm{~K}$ transition temperature of the film is chosen to accommodate operation from a $300 \mathrm{mK}$ bath temperature, as is planned for BLAST. The devices are feedhorn-coupled to a variable temperature blackbody source. Feedhorn-coupling is a standard approach at sub-millimeter wavelengths that has recently been demonstrated with MKIDs. ${ }^{20}$ Our measurements show traits associated with photon-noise limited detection in MKIDs. The NEP scales as $\sqrt{P}$, and the noise spectra are flat with a roll-off set by quasiparticle recombination and resonator ring-down time.

Figure 1 shows the detector array package, detector design, and feedhorn-coupled mounting scheme. We grow a 


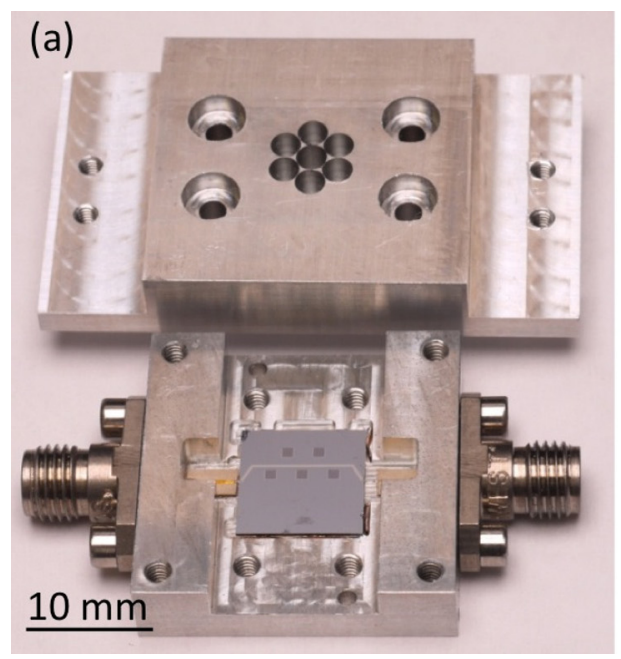

(b)

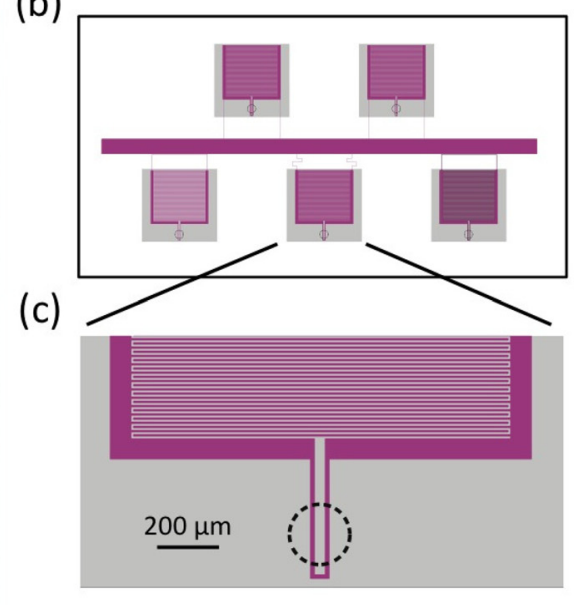

(d)

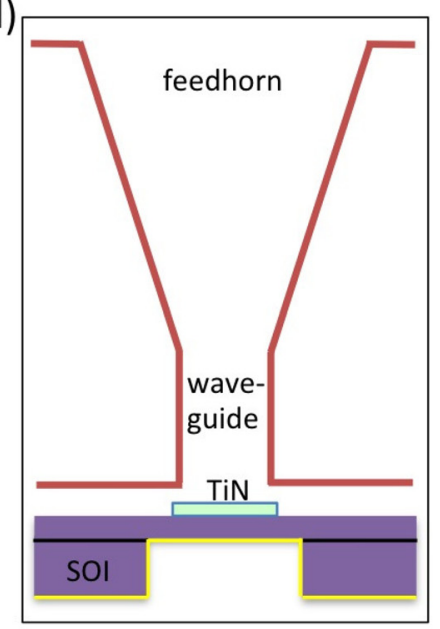

FIG. 1. Detector package and optical coupling. (a) Photograph of 5-pixel die mounted in a sample holder together with the feedhorn array. (b) Detector chip design showing that the cross-wafer microstrip feedline couples to five lumped-element resonators. The grey squares indicate where backside silicon is removed from the SOI wafer to create the quarter-wavelength backshort. (c) Zoom-in view of a single pixel comprised of a $5 \mu \mathrm{m}$ IDC and single turn inductor. The dashed circle locates the position of the feedhorn's exit waveguide, the optically active region. (d) Cross-section schematic (not to scale) of the feedhorn/ waveguide fed coupling scheme, which illustrates the silicon membrane backshort.

4/10/4 nm thick trilayer film of TiN/Ti/TiN as described in Ref. 21 on a silicon-on-insulator (SOI) wafer. The film is patterned into five $f \sim 1 \mathrm{GHz}$ lumped-element resonators each comprised of a $5 \mu \mathrm{m}$ width and spacing interdigitated capacitor (IDC) of total area $0.9 \mathrm{~mm}^{2}$ in parallel with a oneturn inductor. The width of the inductor strip is $8 \mu \mathrm{m}$, and has a total volume $V=86 \mu \mathrm{m}^{3}$. The resonators on the chip couple to a $50 \Omega$ cross-wafer microstrip feedline, also made of the trilayer. We measure $T_{c}=1.4 \mathrm{~K}$, internal quality factors from 200000 to 400,000 at bath temperature $T_{\text {bath }}=75$ $\mathrm{mK}$, and coupling quality factors $\sim 30000$.

The inductor element also acts as the absorber of incident sub-millimeter radiation. It is located at $\sim 50 \mu \mathrm{m}$ below the $173.7 \pm 1 \mu \mathrm{m}$ diameter waveguide output of the feedhorn. The sheet impedance $(90 \Omega / \square)$ of the inductor is matched to the waveguide impedance and absorbs radiation polarized along the long axis of the inductor. The silicon behind the inductor and capacitor is removed with a deep reactive ion etch up to the buried insulator layer of the SOI wafer. This produces a $19 \mu \mathrm{m}$ thick silicon membrane. The oxide layer is removed with a $\mathrm{CHF}_{3} / \mathrm{O}_{2}$ plasma etch. $\mathrm{RF}$ sputter deposition of a $500 \mathrm{~nm}$ thick layer of $\mathrm{Nb}$ on the backside of the wafer creates a quarter-wavelength reflective backshort as well as a continuous ground plane.

We mount this package to the $50 \mathrm{mK}$ stage of an adiabatic demagnetization refrigerator (ADR) cryostat. The feedhorns view a temperature controlled $\mathrm{THz}$ tessellating tile that has $<-30 \mathrm{~dB}$ reflection at $600 \mathrm{GHz}{ }^{22}$ Using the simulated beam profile of the feedhorns, we calculate $>99 \%$ of the optical throughput goes to the blackbody. The tile is glued into a copper block that is weakly linked to the $3 \mathrm{~K}$ stage of the cryostat. We control the load temperature between $4 \mathrm{~K}$ and $21 \mathrm{~K}$ to $\sim 1 \mathrm{mK}$ stability by use of a heater and calibrated thermometer.

The optical passband is defined by the $\nu_{1}=1 \mathrm{THz}$ waveguide cut-off in the feedhorn and a well-characterized 1.4 $\mathrm{THz}$ low-pass filter ${ }^{23}$ mounted to the feedhorn array. The inband power emitted from the load is

$$
P=\int_{\nu} d \nu\left(\frac{c}{\nu}\right)^{2} B(\nu, T) F(\nu),
$$

where we have assumed single-mode optical throughput. $B(\nu, T)$ is the Planck function. The passband $F(\nu)$ includes the measured filter transmission and a calculation of the waveguide cut-off. Metal-mesh filters are known to have harmonic leaks at frequencies above cut-off. ${ }^{24}$ However, the integrated power above the passband is $<2 \%$ of the total inband power, even at the highest blackbody temperatures.

We perform a frequency sweep and characterize noise with a homodyne measurement and a SiGe amplifier at thermal loads ranging from $P=0.3 \mathrm{fW}$ to $21 \mathrm{pW}$ and at $T_{\text {bath }}=75 \mathrm{mK}$. A fit to the complex transmission $\mathrm{S}_{21}(f)$ yields the resonant frequency as a function of thermal power. The fractional frequency shift $\delta f / f$ response of a detector to the changing thermal load is shown in Fig. 2(a). In a separate experiment, we confirm there is no stray coupling to the thermal source by blocking all horn apertures with reflective tape and observing no frequency shift to applied thermal load.

For each thermal load, we measure noise at the microwave frequency that maximizes $\delta S_{21} / \delta f$. The power on the feedline is in a range of $-97 \mathrm{dBm} \lesssim P_{g} \lesssim-87 \mathrm{dBm}$, chosen to be at least $3 \mathrm{~dB}$ below bifurcation to the strong non-linear effects in the resonator. ${ }^{25,26} \mathrm{We}$ project the raw in-phase and quadrature components of the data into the frequency and dissipation quadratures $^{25}$ and examine the noise in the frequency quadrature.

The spectrum of a photon-noise limited MKID is expected to be of Lorentzian form. Example spectra at various thermal loads are shown in Fig. 2(b). When $P>0.5 \mathrm{pW}$, each spectrum is well described by a Lorentzian function of white noise level $A$ and time constant $\tau$ that is summed with a background noise floor $B$

$$
S_{\delta f / f}(\omega)=\frac{A}{1+\omega^{2} \tau^{2}}+B
$$



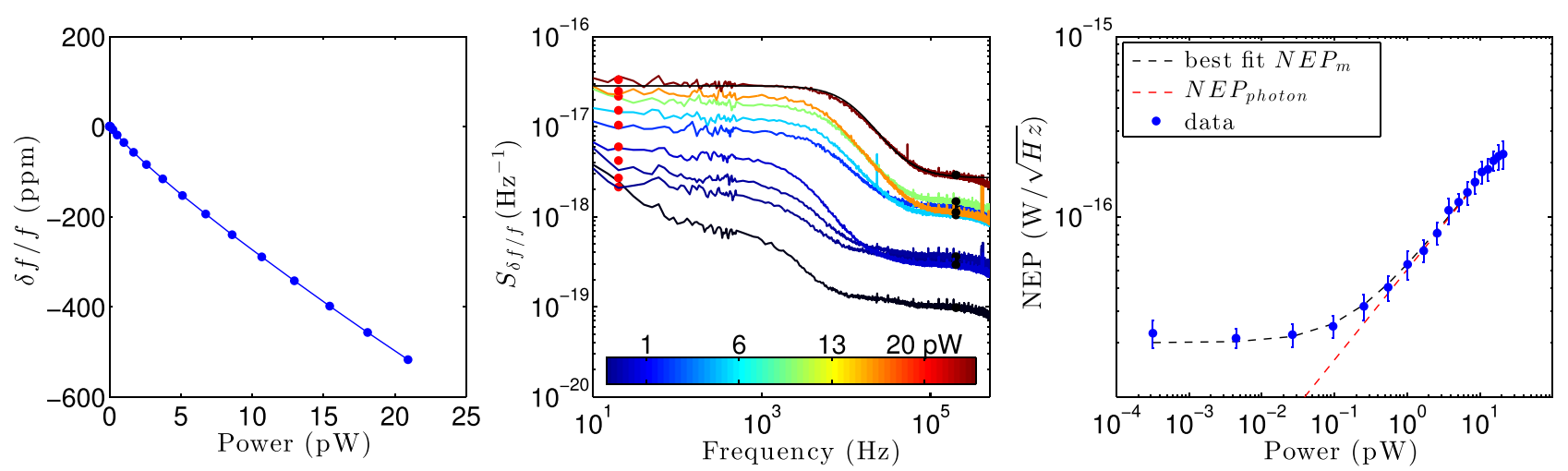

FIG. 2. (a) Fractional frequency response of detector to thermal power emitted by the blackbody load. (b) Noise spectra, in fractional frequency units, for blackbody loads between $4 \mathrm{~K}$ and $21 \mathrm{~K}$ taken at a bath temperature of $75 \mathrm{mK}$. The solid line is an example of Lorentzian model fit to the $P=20 \mathrm{pW}$ data. (c) NEP versus thermal load. The low frequency noise ( $20 \mathrm{~Hz}$, red points) from the spectra in panel (b), corrected for system noise using the $200 \mathrm{kHz}$ black points, is converted to NEP by use of the local slope in (a). These data are in good agreement with the photon-noise prediction (the red, dashed line) at power levels $>0.5 \mathrm{pW}$. The black, dashed line is the best fit NEP model, which includes contributions due to photon noise, recombination noise, and a noise term independent of optical power.

An example fit to the $P=20 \mathrm{pW}$ spectrum is shown as the solid, black line in Fig. 2(b). We observe that the white noise level increases with applied thermal power.

The detector NEP may be determined by dividing the square root of the detector noise in Fig. 2(b) by the local responsivity $\frac{\delta f / f}{\delta P}(P)$ in Fig. 2(a). We estimate the detector noise by subtracting the amplifier noise contribution (black dots at $200 \mathrm{kHz}$ in Fig. 2(b)) from the average noise at $20 \mathrm{~Hz}$ (red dots in Fig. 2(b)), which is determined by a power law fit to the data around $20 \mathrm{~Hz}$. These values, together with one standard deviation fit uncertainty, are plotted as a function of thermal load power $P$ in Fig. 2(c), which is the main result of this letter. Above $0.5 \mathrm{pW}$, the measured NEP scales as $\sqrt{P}$, as expected for a photon-noise limited detector. Furthermore, the red, dashed line of Fig. 2(c) is the photon noise prediction, calculated using Eq. (1) $(\nu=1.25 \mathrm{THz}$, $\eta=0.69$, and $m<0.1$ for all blackbody temperatures). For $P \geq 0.5 \mathrm{pW}$, the data are in good agreement with the photonnoise prediction.

The full data set fits the model (dashed-black line of Fig. 2(c))

$$
N E P_{m}^{2}=N E P_{\alpha}^{2}+\frac{N E P_{\text {photon }}^{2}+N E P_{R}^{2}}{\eta},
$$

which has been used to determine the optical efficiency $\eta{ }^{11}$ $\left(N E P_{\alpha}\right.$ is a constant term, independent of optical power and discussed below). The detector responds to both co- and cross-polar radiation as $\delta f \propto \eta_{c} P_{c}+\eta_{x} P_{x}$, where $\eta_{c}\left(\eta_{x}\right)$ and $P_{c}\left(P_{x}\right)$ are the efficiency and power emitted in the co- (cross-) polar component, respectively. The frequency responsivity and NEP we report are referred to the power in a single polarization from a source with equal power in both polarizations, as is the case for the thermal source used in our experiment. As a result of this description, $\eta=\eta_{c}+\eta_{x}$. The fit yields $\eta=0.69 \pm 0.01$, which is lower than the sum $\eta_{c}+\eta_{x}$ $=0.64+0.16=0.80$ determined from electromagnetic coupling simulations, which assume a $\Delta Z=50 \mu \mathrm{m}$ air gap between the absorber and waveguide. The discrepancy may be due to uncertainty in the positioning of the absorber with respect to the waveguide output or absorption in the aluminum feedhorn, which has not been accounted for. To pin down $\eta_{c}$ and $\eta_{x}$ separately requires measurements with a polarizer. The design and measured polarization performance of this detector type is the subject of a future publication. For polarimetry applications, such as BLAST, ${ }^{19} \eta_{c} \gg \eta_{x}$ is required. By splitting the absorber width into $2 \mu \mathrm{m}$ strips, simulations suggest that $\eta_{c} \sim 0.8$ and $\eta_{x} \lesssim 0.02$ are achievable.

At $P<0.1 \mathrm{pW}$, we find that the basic shape of the spectra are unchanged and that the NEP saturates to $N E P_{\alpha}=2 \times 10^{-17} \mathrm{~W} / \sqrt{\mathrm{Hz}}$. Initial measurements suggest that the source of this noise is from a background of excess quasiparticles due to stray light absorption. We will conduct future tests in a carefully designed light-tight box to confirm that $N E P_{\alpha}$ decreases when stray light is reduced. However, the current value of $N E P_{\alpha}$ has a small effect on the sensitivity for many potential applications.

Finally, we discuss the implications of the near constant responsivity to increased thermal loading (Fig. 2(a)). This phenomenon has previously been observed in TiN films ${ }^{15,27}$ and departs from the behavior in conventional superconducting materials, which show $\delta f / \delta P \sim P^{-0.5}$ as a consequence of (1) $\delta f \propto \delta n_{q p}$ and (2) $P \propto n_{q p}^{2}$. Here, $n_{q p}$ is the quasiparticle number density. Our linear responsivity measurement suggests that (1), (2), or both relationships are not valid for our TiN trilayer films. If $\delta f \propto \delta n_{q p}$ holds, the observed constant responsivitiy suggests $P \propto n_{q p}$, which further implies that the quasiparticle lifetime time $\tau_{q p}$ is independent of $P$. However, the lifetimes inferred from the increasing roll-off in the noise is inconsistent with this picture, which may indicate that relationship (1) does not hold for this material. This apparent discrepancy remains outstanding and requires future investigation. Understanding the recombination physics of TiN films is a key in explaining the observed linear responsivity. We plan to investigate this device physics by directly measuring the detector response to optical pulses in future experiments.

In conclusion, we have demonstrated photon-noise limited sensitivity in feedhorn-coupled microwave kinetic inductance detectors fabricated from a superconducting TiN/ $\mathrm{Ti} / \mathrm{TiN}$ trilayer film. This work represents a significant step 
towards realizing high sensitivity, large-format kinetic inductance detector arrays suitable for broadband photometry, and with polarimetric sensing capability. Further work is planned to explore the origin of $N E P_{\alpha}$ and to better understand the anomalous quasiparticle physics in our TiN/Ti/TiN films.

This work was supported in part by NASA through Grant No. NNX13AE50G S04. TiN materials research was supported in part by DARPA. Brad Dober is supported by the NASA Earth and Space Science Fellowship. The authors would like to thank Edward Wollack and Lev Ioffe for useful discussions.

${ }^{1}$ P. K. Day, H. G. LeDuc, B. A. Mazin, A. Vayonakis, and J. Zmuidzinas, Nature 425, 817 (2003).

${ }^{2}$ J. Baselmans, J. Low. Temp. Phys. 167(3-4), 292 (2012).

${ }^{3}$ S. R. Golwala, C. Bockstiegel, S. Brugger, N. G. Czakon, P. K. Day, T. P. Downes, R. Duan, J. Gao, A. K. Gill, J. Glenn et al., SPIE Proc. 8452, 845205 (2012).

${ }^{4}$ L. J. Swenson, P. K. Day, C. D. Dowell, B. H. Eom, M. I. Hollister, R. Jarnot, A. Kovács, H. G. Leduc, C. M. McKenney, R. Monroe et al., SPIE Proc. 8452, 84520P (2012).

${ }^{5}$ E. Shirokoff, P. S. Barry, C. M. Bradford, G. Chattopadhyay, P. Day, S. Doyle, S. Hailey-Dunsheath, M. I. Hollister, A. Kovács, C. McKenney et al., SPIE Proc. 8452, 84520R (2012).

${ }^{6}$ A. Monfardini, R. Adam, A. Adane, P. Ade, P. André, A. Beelen, B. Belier, A. Benoit, A. Bideaud, N. Billot et al., J. Low. Temp. Phys. 176, 787 (2014)

${ }^{7}$ J. Hubmayr, J. Beall, D. Becker, J. Brevik, H. Cho, G. Che, M. Devlin, B. Dober, J. Gao, N. Galitzki et al., J. Low. Temp. Phys. 176(3-4), 490 (2014).

${ }^{8}$ J. Zmuidzinas, Appl. Opt. 42, 4989 (2003).

${ }^{9}$ P. J. de Visser, J. J. A. Baselmans, P. Diener, S. J. C. Yates, A. Endo, and T. M. Klapwijk, Phys. Rev. Lett. 106(16), 167004 (2011).

${ }^{10}$ C. Wilson, L. Frunzio, and D. Prober, Phys. Rev. Lett. 87(6), 067004 (2001).

${ }^{11}$ S. J. C. Yates, J. J. A. Baselmans, A. Endo, R. M. J. Janssen, L. Ferrari, P. Diener, and A. M. Baryshev, Appl. Phys. Lett. 99(7), 073505 (2011).
${ }^{12}$ T. Guruswamy, D. Goldie, and S. Withington, Supercond. Sci. Technol. 27(5), 055012 (2014).

${ }^{13}$ P. J. de Visser, J. J. A. Baselmans, J. Bueno, N. Llombart, and T. M. Klapwijk, Nat. Commun. 5, 3130 (2014).

${ }^{14}$ R. M. J. Janssen, J. J. A. Baselmans, A. Endo, L. Ferrari, S. J. C. Yates, A. M. Baryshev, and T. M. Klapwijk, Appl. Phys. Lett. 103(20), 203503 (2013).

${ }^{15}$ C. M. McKenney, H. G. Leduc, L. J. Swenson, P. K. Day, B. H. Eom, and J. Zmuidzinas, SPIE Proc. 8452, 84520S (2012).

${ }^{16}$ H. G. Leduc, B. Bumble, P. K. Day, B. H. Eom, J. Gao, S. Golwala, B. A. Mazin, S. McHugh, A. Merrill, D. C. Moore et al., Appl. Phys. Lett. 97(10), 102509 (2010).

${ }^{17}$ M. R. Vissers, J. Gao, D. S. Wisbey, D. A. Hite, C. C. Tsuei, A. D. Corcoles, M. Steffen, and D. P. Pappas, Appl. Phys. Lett. 97(23), 232509 (2010).

${ }^{18}$ M. R. Vissers, J. Gao, J. S. Kline, M. Sandberg, M. P. Weides, D. S. Wisbey, and D. P. Pappas, Thin Solid Films 548, 485 (2013).

${ }^{19}$ N. Galitzki, P. Ade, F. E. Angile, P. Ashton, J. Beall, D. Becker, K. J. Bradford, G. Che, H.-M. Cho, M. J. Devlin et al., J. Astron. Instrum. 3(02), 1440001 (2014).

${ }^{20}$ H. McCarrick, D. Flanigan, G. Jones, B. Johnson, P. Ade, D. Araujo, K. Bradford, R. Cantor, G. Che, P. Day et al., Rev. Sci. Instrum. 85(12), 123117 (2014).

${ }^{21}$ M. R. Vissers, J. Gao, M. Sandberg, S. M. Duff, D. S. Wisbey, K. D. Irwin, and D. P. Pappas, Appl. Phys. Lett. 102(23), 232603 (2013).

${ }^{22}$ J. Saily and A. V. Raisanen, "Studies on specular and non-specular reflectivities of radar absorbing materials (RAM) at submillimetre wavelengths," Tech. Rep., Report No. S 258, Radio Laboratory, Department of Electrical and Communications Engineering, Helsinki University of Technology, 2003.

${ }^{23}$ E. Pascale, P. A. R. Ade, J. J. Bock, E. L. Chapin, J. Chung, M. J. Devlin, S. Dicker, M. Griffin, J. O. Gundersen, M. Halpern et al., Astrophys. J. 681, 400 (2008).

${ }^{24}$ P. A. R. Ade, G. Pisano, C. Tucker, and S. Weaver, SPIE Proc. 6275, $62750 \mathrm{U}$ (2006).

${ }^{25}$ J. Zmuidzinas, Annu. Rev. Condens. Matter Phys. 3(1), 169 (2012).

${ }^{26}$ L. J. Swenson, P. K. Day, B. H. Eom, H. G. Leduc, N. Llombart, C. M. McKenney, O. Noroozian, and J. Zmuidzinas, J. Appl. Phys. 113(10), 104501 (2013).

${ }^{27}$ O. Noroozian, "Superconducting microwave resonator arrays for submillimeter/far-infrared imaging," Ph.D. dissertation (California Institute of Technology, 2012). 